\title{
Phase I study of FOLFIRI plus pimasertib as second-line treatment for KRAS-mutated metastatic colorectal cancer
}

\author{
T Macarulla ${ }^{1}$, A Cervantes $^{2}$, J Tabernero ${ }^{1}$, S Roselló ${ }^{2}$, E Van Cutsem ${ }^{3}$, S Tejpar $^{3}$, H Prenen $^{3}$, E Martinelli $^{4}$,

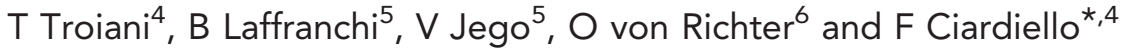 \\ ${ }^{1}$ Vall d'Hebron University Hospital and Institute of Oncology (VHIO), Universitat Autònoma de Barcelona, P Vall d'Hebron 119-129, \\ Barcelona 08035, Spain; ${ }^{2}$ Department of Haematology and Medical Oncology, Biomedical Research Institute INCLIVA, University \\ of Valencia, Avda Blasco Ibáñez 17, Valencia 46010, Spain; ${ }^{3}$ Department of Gastroenterology, University Hospitals Gasthuisberg \\ and KU Leuven, Herestraat 49, Leuven 3000, Belgium; ${ }^{4}$ Department of Experimental and Clinical Medicine, Second University of \\ Naples, Via S Pansini 5, Naples 80131, Italy; ${ }^{5}$ Merck Serono SA, 9 Chemin des Mines, Geneva 1202, Switzerland and ${ }^{6}$ Merck KGaA, \\ Frankfurter Strasse 250, F131/102, Darmstadt 64293, Germany
}

Background: The mitogen-activated protein kinase (MAPK) pathway has been implicated in the molecular pathogenesis of human cancers, including metastatic colorectal cancer (mCRC). This provides a rationale for the development of MAPK-targeted agents such as pimasertib.

Methods: Patients with KRAS mutant $\mathrm{mCRC}$ were treated in the second-line setting with FOLFIRI (5-fluorouracil/folinic acid/ irinotecan) plus pimasertib. The primary objective of the safety run-in phase was to determine the maximum-tolerated dose (MTD) and the recommended phase II dose of pimasertib combined with FOLFIRI.

Results: Sixteen patients were enrolled in the trial. Ten and six patients were treated daily with 45 and $60 \mathrm{mg}$ of pimasertib plus FOLFIRI, respectively. The MTD was considered to be $45 \mathrm{mg}$ per day. The most common treatment-emergent adverse events were diarrhoea, nausea, vomiting, asthenia and skin/rash event. Of the 15 patients in the efficacy analysis group, two patients had partial response, nine patients had stable disease, three patients had progressive disease as their best overall response and one patient could not be evaluated.

Conclusions: Dose escalation of pimasertib in combination with FOLFIRI was limited by toxicity. At the MTD of 45 mg per day, pimasertib was adequately tolerated in patients with $\mathrm{MCRC}$ and no unexpected or new safety signals or concerns were identified.

The mitogen-activated protein kinase (MAPK) (RAS/RAF/MEK/ ERK) pathway has a central role in cell-cycle regulation, cell growth, signalling and survival. The MAPK pathway is frequently dysregulated and aberrantly activated in human cancers, providing a strong rationale for developing targeted therapies aimed at blocking key steps of the pathway (Sebolt-Leopold, 2008; Fremin and Meloche, 2010; Santarpia et al, 2012). The development of MEK inhibitors that target effectors with activity downstream of KRAS may be particularly important for tumours where KRAS is constitutively activated as a result of mutation because they offer the potential to expand therapeutic options.

KRAS mutations are common in metastatic colorectal cancer (mCRC), occurring in $\sim 40 \%$ of patients (Neumann et al, 2009; De Roock et al, 2011; Van Cutsem et al, 2011). KRAS status influences treatment options for patients with mCRC (Van Cutsem et al, 2010; Schmoll et al, 2012) and this has been well documented in the case of epidermal growth factor receptor inhibitors, which are not clinically effective in KRAS mutant (mt) mCRC

*Correspondence: Professor F Ciardiello; E-mail: fortunato.ciardiello@unina2.it

Received 30 June 2014; revised 25 February 2015; accepted 25 March 2015; published online 19 May 2015

(c) 2015 Cancer Research UK. All rights reserved 0007-0920/15 
(Bokemeyer et al, 2009, 2012; Van Cutsem et al, 2009, 2011; De Roock et al, 2010; Douillard et al, 2010, 2013; Ye et al, 2013). First-line treatment options for patients with KRAS mt mCRC are 5-fluorouracil (5-FU)/folinic acid (FA)/oxaliplatin (FOLFOX), capecitabine/oxaliplatin (XELOX) or 5-FU/FA/irinotecan (FOLFIRI), with or without the vascular endothelial growth factor inhibitor, bevacizumab (Van Cutsem et al, 2010; Schmoll et al, 2012). The therapeutic options for patients with KRAS mt mCRC who fail first-line treatment with combination chemotherapy plus bevacizumab are limited and thus there is an unmet need for new efficacious agents in the second-line setting for this patient population (Prenen et al, 2010).

Pimasertib (MSC1936369B/AS703026) is a highly selective, orally bioavailable, small-molecule inhibitor of the MEK1/2 kinases of the MAPK pathway, and has demonstrated potent antitumour activity either alone or in combination with other agents in cell lines and xenograft models (Clark et al, 2009; Goutopoulos et al, 2009; Machl et al, 2009; Kim et al, 2010; Yoon et al, 2011; Martinelli et al, 2013). A phase I dose-escalation trial revealed dose-limiting toxicities of skin rash, acneiform dermatitis, ocular events and stomatitis (Awada et al, 2012). This trial also showed that the median plasma half-life was $5 \mathrm{~h}$ and that optimal target suppression and ocular safety were achieved with twice-daily continuous dosing of pimasertib $60 \mathrm{mg}$. Therefore, a recommended single-agent pimasertib dose of $60 \mathrm{mg}$ twice-daily administered continuously was selected for phase II trials. Pimasertib is currently undergoing phase I/II clinical trials in patients with a range of tumour types and has demonstrated clinical activity both as monotherapy and in combination with other agents (Ravandi et al, 2011; Delord et al, 2012; Infante et al, 2012; Naing et al, 2012).

A two-part, open-label, multicentre study, comprising a safety run-in part followed by a randomised phase II part, was designed to investigate the safety and efficacy of adding pimasertib to FOLFIRI as second-line treatment for patients with KRAS mt mCRC. Here, we report the results of the safety run-in part of this study.

\section{MATERIALS AND METHODS}

Study objectives. The primary objective of the safety run-in phase was to determine the maximum-tolerated dose (MTD) and the recommended phase II dose (RP2D) of pimasertib combined with FOLFIRI. Secondary objectives included assessment of the pharmacokinetics (PK) of pimasertib and irinotecan and evaluation of the antitumour activity of FOLFIRI combined with pimasertib as second-line treatment for patients with KRAS mt mCRC.

Patient eligibility. Eligible patients were aged $\geq 18$ years with histologically confirmed KRAS mt mCRC with disease progressing during or following first-line treatment for metastatic disease with oxaliplatin plus fluoropyrimidine-based chemotherapy (with or without bevacizumab). Patients were required to have measurable metastatic disease according to Response Evaluation Criteria In Solid Tumours (RECIST v1.0), an Eastern Cooperative Oncology Group performance status of 1 or less and adequate hepatic (total bilirubin $<1.5 \times$ upper limit of normal (ULN), or aspartate aminotransferase (AST)/alanine aminotransferase (ALT) $<2.5 \times \mathrm{ULN}$ (for subjects with liver involvement, AST/ ALT $<5 \times$ ULN)), renal (serum creatinine $<1.5 \times$ ULN and/or calculated creatinine clearance (Cockroft-Gault formula) $>50 \mathrm{ml} \mathrm{min}^{-1}$ ) and marrow function (haemoglobin $>9.0 \mathrm{~g} \mathrm{dl}^{-1}$, neutrophil count $>1.5 \times 10^{9} 1^{-1}$ and/or platelet count $\left.>100 \times 10^{9} 1\right)$. Main exclusion criteria comprised retinal degenerative disease and history of uveitis or retinal vein occlusion. Patients should have not received chemotherapy with any investigational drug or participated in another clinical trial within 4 weeks before study treatment. Enrolled patients were not allowed to have received radiotherapy on more than $30 \%$ of bone marrow reserves or bone marrow/stem cell transplantation before study treatment.

Trial design and treatment schedule. This was a two-part, openlabel, multicentre study (four European centres; two in Spain, one in Belgium and one in Italy), comprising a safety run-in part followed by a randomised phase II part. Treatment comprised 28-day cycles of FOLFIRI and pimasertib. Patients received FOLFIRI on days 1 and 15 of each cycle. FOLFIRI comprised irinotecan $\left(180 \mathrm{mg} \mathrm{m}^{-2}\right)$ and FA (1-leucovorin/FA $200 \mathrm{mg} \mathrm{m}^{-2}$ or DL-leucovorin $400 \mathrm{mg} \mathrm{m}^{-2}, 90$-min infusion), followed by 5 -FU ( $400 \mathrm{mg} \mathrm{m}^{-2}$ bolus then $2400 \mathrm{mg} \mathrm{m}^{-2} 46-\mathrm{h}$ infusion). Pimasertib, at a starting dose of $45 \mathrm{mg}$ per day, was administered orally on a 5-day on/2-day off schedule continuously during the cycle (days $1-5,8-12,15-19$ and 22-26). Beyond cycle 1, patients were allowed to continue the trial treatment until progressive disease, intolerable toxicity or investigator/patient decision. The study protocol was approved by independent ethics committees and met all legal and regulatory requirements. The study was conducted in accordance with the Declaration of Helsinki and all patients provided written, informed consent.

Pimasertib dose escalation. The study used a standard $3+3$ cohort design, with escalation of pimasertib dose from $45 \mathrm{mg}$ per day in $\sim 33 \%$ increments until the MTD was reached. Dose escalation of pimasertib was based on the occurrence of defined dose-limiting toxicities (DLTs) during cycle 1 of therapy. The MTD was the dose immediately below the dose associated with more than one DLT. The MTD cohort was to be expanded to nine patients to gather more safety information at this dose level. Doselimiting toxicities were monitored centrally and the decision to escalate to the next dose level was taken by a safety monitoring committee.

Safety. Dose-limiting toxicities were classified according to the National Cancer Institute Common Terminology Criteria for Adverse Events v.3.0 and defined as toxicities possibly or probably related to trial treatments. Dose-limiting toxicities comprised the following: any non-haematological toxicity of grade 3 or more (excluding asymptomatic increases in liver function tests reversible within 7 days and adequately controlled grade 3 vomiting or diarrhoea); any grade 4 neutropenia of more than 5 days duration or febrile neutropenia lasting more than 1 day; grade 4 thrombocytopenia (grade 3 if accompanied by bleeding); and any treatment delay of more than 2 weeks owing to trial treatmentrelated adverse events (AEs). Safety parameters in addition to DLT determination included AEs, deaths, clinical laboratory abnormalities, vital signs and ophthalmologic assessments.

Pharmacokinetics. Plasma samples for PK analysis of pimasertib, irinotecan/SN-38 and 5-FU were collected at three timepoints during cycle 1: (i) following administration of single doses of pimasertib and FOLFIRI on day 1; (ii) following administration of a single dose of pimasertib on day 8; and (iii) following administration of a single dose of FOLFIRI on day 15.

Analysis of pimasertib on day 8, following a 2-day period without pimasertib administration based on the 5-day on/2-day off schedule, was sufficient to wash out the pimasertib administered on days 1-5 and all FOLFIRI components administered on day 1. This was used to characterise pimasertib single-dose PK in patients with mCRC. An intraindividual crossover comparison of these data with the PK of single doses of pimasertib in the presence of FOLFIRI, assessed using samples taken on day 1, was performed to evaluate any effect of FOLFIRI on the PK of pimasertib. The PK of pimasertib at steady state was not assessed in this study. 
For the evaluation of the $\mathrm{PK}$ of irinotecan and its active metabolite (SN-38) in the presence (day 1) and absence of pimasertib (day 15), plasma samples were collected following a $180 \mathrm{mg} \mathrm{m}^{-2}$ irinotecan infusion on days 1 and 15 , respectively. To ensure the absence of pimasertib on day 15, pimasertib dosing on days 15-19 of cycle 1 was shifted to days 16-20, allowing a 2-day wash-out period following pimasertib administration on days 8-12. Intraindividual comparisons were performed to elucidate the effect of single doses of pimasertib on $\mathrm{PK}$ of irinotecan and SN-38. Pimasertib, irinotecan and SN-38 plasma concentrations were determined using validated HPLC-MS/MS methods.

5-fluorouracil concentrations were determined in irinotecan back-up samples for subjects receiving a 46-h infusion in the presence (day 1) and absence (day 15) of pimasertib. The samples were scheduled for 6,8 and $24 \mathrm{~h}$ after the start of irinotecan infusion. These times corresponded to $\sim 4,6$ and $22 \mathrm{~h}$ after the start of the 46-h 5-FU infusion, respectively. The mean apparent terminal half-life of 5 -FU following intravenous administration is $\sim 16$ min (range $8-20 \mathrm{~min}$ ). The systemic concentration reaches steady state after 5 half-lives (in this case after about $1.5 \mathrm{~h}$ ). Therefore, the selected time points for 5-FU blood sampling represented steady-state concentrations, which are under the influence of varying infusion rates by the infusion pump or effect of concomitantly administered pimasertib. Only a subset of patients to whom 5-FU was administered using an electronic pump was analysed, as the variability in 5-FU steady-state concentrations introduced by other infusion techniques was considered too high to detect possible effects of pimasertib on 5 -FU steady-state concentrations. Intraindividual ratios of 5-FU concentrations were calculated to assess the impact of pimasertib on the PK of 5-FU.

Pharmacokinetic parameters were evaluated at Merck Serono (Darmstadt, Germany) according to standard non-compartmental methods using the computer program KINETICA, version 4.4.1.

Efficacy. Target and non-target lesions were evaluated at baseline, every 6 weeks and at the end of treatment by computed tomography (CT) or magnetic resonance imaging (if the patient had contraindications to CT) according to RECIST v.1.0. The best overall response for each patient was defined as the best response recorded from the start of treatment until disease progression using RECIST v.1.0.

Statistics. All data analyses for the safety run-in phase of the trial were descriptive. The DLT analysis set included all patients who experienced a DLT and those who did not experience a DLT but received at least $90 \%$ of the full planned dose of pimasertib (i.e., 18 days out of 20 planned) during the first cycle. These patients should have also received at least two FOLFIRI infusions during the first cycle independent of whether there were dose delays or not. Safety data were collected during the first cycle. The safety analysis set included all patients who received at least one administration of trial medication (any of the three FOLFIRI components and pimasertib). The efficacy analysis set included all patients who received at least one administration of trial medication (any of the three FOLFIRI components and pimasertib) and had a baseline tumour assessment and at least one postbaseline efficacy assessment.

\section{RESULTS}

Patient disposition. A total of 16 patients were recruited from March 2010 to May 2012. All patients received study treatment and were evaluable for safety. Owing to permanent discontinuation (consent withdrawal, day 12 of cycle 1), one patient (treated with pimasertib $45 \mathrm{mg}$ per day) was excluded from the dose-escalation,
DLT and efficacy analyses $(n=15)$. All patients had at least one evaluable plasma concentration-time profile, and therefore were included in the PK analysis set. All treated patients were off treatment by November 2012. The major reason for patients ending treatment was disease progression $(n=8)$; other reasons included withdrawal of consent $(n=3)$ and AEs $(n=2)$.

Patient baseline characteristics. Baseline demographic and clinical characteristics are shown in Table 1 . Ten patients $(62.5 \%)$ had adenocarcinoma of the colon and six patients (37.5\%) had adenocarcinoma of the rectum. All 16 patients had received at least one type of prior anticancer therapy. Most patients $(n=12$; $75 \%)$ had received previous chemotherapy for metastatic disease; four had received prior adjuvant chemotherapy and had mCRC at study entry. Thirteen patients $(81.3 \%)$ had received prior surgery and four patients (25\%) had received prior radiotherapy.

Treatment exposure. The median time on trial treatment (FOLFIRI plus pimasertib) was 10.9 weeks (range 1-44 weeks); pimasertib $45 \mathrm{mg}$ per day cohort: 11.0 weeks (range 1-41 weeks)

Table 1. Patient, disease and treatment characteristics at baseline (safety population)

\begin{tabular}{|l|c|c|c|}
\hline Characteristics, $n$ & $\begin{array}{c}\text { Pimasertib } \\
45 \mathrm{mg} \text { per } \\
\text { day }(n=10)\end{array}$ & $\begin{array}{c}\text { Pimasertib } \\
60 \mathrm{mg} \text { per } \\
\text { day }(n=6)\end{array}$ & $\begin{array}{c}\text { Overall } \\
(n=16)\end{array}$ \\
\hline
\end{tabular}

Sex

\begin{tabular}{|l|l|l|l}
\hline Male & 5 & 4 & 9 \\
Female & 5 & 2 & 7
\end{tabular}

\begin{tabular}{|l|c|c|c|}
\hline \multicolumn{4}{|l|}{ Age (years) } \\
\hline Median (range) & $60.6(47-81)$ & $67.6(38-81)$ & $64.0(38-81)$ \\
\hline ECOG PS
\end{tabular}

ECOG PS

\begin{tabular}{|l|c|c|c|}
\hline 0 & 6 & 3 & 9 \\
1 & 4 & 3 & 7 \\
\hline \multicolumn{2}{|l|}{ Location of primary tumour } \\
\hline $\begin{array}{l}\text { Adenocarcinoma of } \\
\text { the colon } \\
\begin{array}{l}\text { Adenocarcinoma of } \\
\text { the rectum }\end{array}\end{array}$ & 6 & 4 & 10 \\
\hline $\begin{array}{l}\text { Median number of } \\
\text { metastatic sites } \\
\text { (range) }\end{array}$ & $2.0(2.0-4.0)$ & $1.5(1.0-2.0)$ & $2.0(1.0-4.0)$ \\
\hline
\end{tabular}

\begin{tabular}{|c|c|c|c|}
\hline \multicolumn{4}{|c|}{ Metastatic sites (n) } \\
\hline Liver & 8 & 5 & 13 \\
\hline Lung & 8 & 3 & 11 \\
\hline Lymph nodes & 5 & 1 & 6 \\
\hline Peritoneum & 1 & 0 & 1 \\
\hline Bone & 1 & 0 & 1 \\
\hline Other & 1 & 0 & 1 \\
\hline \multicolumn{4}{|l|}{ Prior treatment } \\
\hline $\begin{array}{l}\text { CT for metastatic } \\
\text { disease }\end{array}$ & 7 & 5 & 12 \\
\hline Oxaliplatin & 7 & 5 & 12 \\
\hline 5-FU/FA & 7 & 4 & 11 \\
\hline Capecitabine & 0 & 1 & 1 \\
\hline Bevacizumab & 4 & 2 & 6 \\
\hline Other & 1 & 2 & 3 \\
\hline Radiotherapy & 3 & 1 & 4 \\
\hline Surgery & 9 & 4 & 13 \\
\hline Adjuvant $\mathrm{CT}^{\mathrm{a}}$ & 3 & 1 & 4 \\
\hline Oxaliplatin & 3 & 1 & 4 \\
\hline $5-\mathrm{FU}$ & 2 & 1 & 3 \\
\hline Capecitabine & 1 & 0 & 1 \\
\hline Bevacizumab & 1 & 0 & 1 \\
\hline \multicolumn{4}{|c|}{ 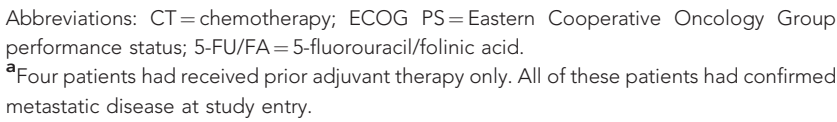 } \\
\hline
\end{tabular}


and pimasertib $60 \mathrm{mg}$ per day cohort: 9.8 weeks (range 5-44 weeks). The median number of initiated treatment cycles was 2.5 (range $1-10$ ), with a median of two cycles (range $0-10$ ) completed. Each cycle was of 4 weeks duration.

Dose-limiting toxicities. Of the 15 patients included in the DLT analysis set, three experienced a DLT. There were no DLTs in the first three patients treated with pimasertib $45 \mathrm{mg}$ per day, allowing dose escalation to pimasertib $60 \mathrm{mg}$ per day in the next cohort. In this cohort, two of six patients receiving pimasertib $60 \mathrm{mg}$ per day had DLTs (grade 3 mucositis in both patients). Thus, the MTD for pimasertib in combination with FOLFIRI was considered to be $45 \mathrm{mg}$ per day. Following per protocol expansion of the $45 \mathrm{mg}$ per day dose level from three to nine patients, one of the six additional patients experienced a DLT (grade 3 hyponatraemia).

Treatment-emergent AEs. All 16 patients had at least one treatment-emergent AE (TEAE), the majority of which were mild or moderate in intensity. The most common types of TEAE were gastrointestinal disorders (diarrhoea: $n=13$; nausea: $n=8$; vomiting: $n=8)$, asthenia $(n=10)$ and skin/rash $(n=8)$. Twelve patients had one or more grade $\geq 3$ TEAEs, most frequently neutropenia (grade $3, n=3$; grade $4, n=2$ ), diarrhoea and mucosal inflammation (both $n=3$, all grade 3 ). There were no TEAEs with a fatal outcome. The three deaths that occurred during the study were all due to disease progression.

Treatment-emergent AEs considered to be related to pimasertib occurred in all patients (Table 2). The most frequent of these were diarrhoea (62.5\% of patients), skin/rash (50\%), asthenia (37.5\%) and serous retinal detachment (SRD; preferred term for detachment of retinal pigment epithelium and macular degeneration)) (37.5\%). The incidence of grade $\geq 3$ pimasertib-related TEAEs was approximately balanced between the two pimasertib dosing cohorts (50.0\% for the $45 \mathrm{mg}$ per day dose and $66.7 \%$ for the $60 \mathrm{mg}$ per day dose). Treatment-emergent AEs of special interest, which are class effects for MEK inhibitors, are shown in Table 3. Seven patients $(43.8 \%)$ reported at least one ocular event during trial treatment. All these events were grade 1, except for a grade 2 SRD reported in one patient. All the ocular events resolved, except for SRD in two patients. Nine patients (56.3\%) experienced skin/rash events (Table 3). Two patients receiving pimasertib $60 \mathrm{mg}$ per day had grade 2 events; all other events were grade 1 . Treatment-emergent AEs considered to be related to FOLFIRI occurred in all patients.

Efficacy. Of the 15 patients in the efficacy analysis set, two (13.3\%) had a partial response (PR; one with pimasertib $45 \mathrm{mg}$ per day and one with pimasertib $60 \mathrm{mg}$ per day), nine $(60.0 \%)$ had stable disease (SD) and three (20.0\%) had progressive disease as their best overall response. Best overall response status was not evaluable for the remaining patient. Stable disease was maintained for at least 12 weeks in six of nine (66.7\%) patients (pimasertib $45 \mathrm{mg}$ per day $(n=5)$ and pimasertib $60 \mathrm{mg}$ per day $(n=1))$.

Pharmacokinetics. In the absence of FOLFIRI, pimasertib was rapidly absorbed, with median $T_{\max }$ values in the 45 and $60 \mathrm{mg}$ per day cohorts of 1.5 and $1.25 \mathrm{~h}$, respectively. Maximum plasma concentrations decreased in a biphasic manner, that is, rapidly up

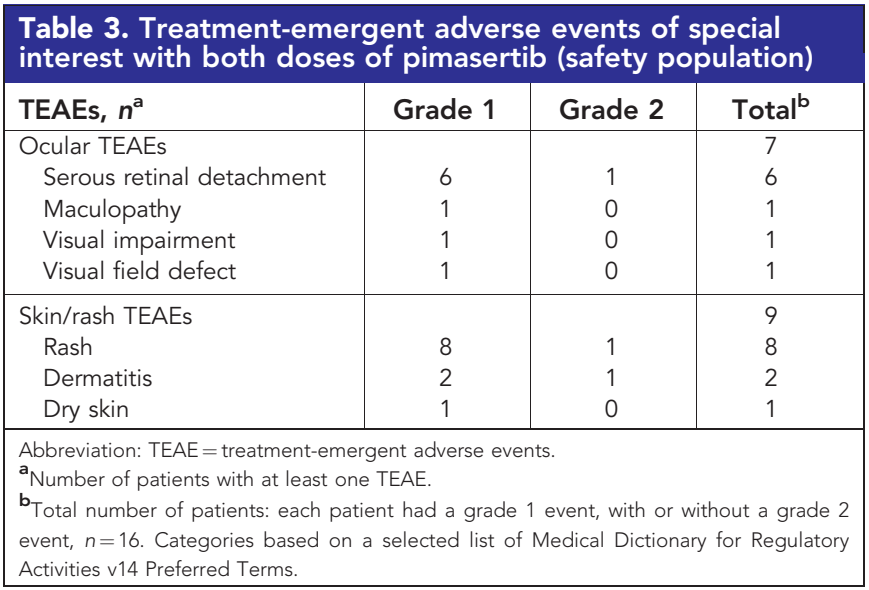

Table 2. Pimasertib-related treatment-emergent adverse events by worst grade (occurring in $\geq 2$ patients overall (all grades) by preferred term): safety population

\begin{tabular}{|c|c|c|c|c|c|c|c|c|c|}
\hline \multirow[b]{3}{*}{ System organ class preferred term } & \multicolumn{3}{|c|}{ Pimasertib $45 \mathrm{mg}$ per day $(n=10)$} & \multicolumn{3}{|c|}{ Pimasertib $60 \mathrm{mg}$ per day $(n=6)$} & \multicolumn{3}{|c|}{ Overall $(n=16)$} \\
\hline & \multicolumn{3}{|c|}{ Grade } & \multicolumn{3}{|c|}{ Grade } & \multicolumn{3}{|c|}{ Grade } \\
\hline & $1 / 2$ & 3 & 4 & $1 / 2$ & 3 & 4 & $1 / 2$ & 3 & 4 \\
\hline Patients with $\geq 1$ TEAE (by worst grade) & 5 & 5 & 0 & 2 & 2 & 2 & 7 & 7 & 2 \\
\hline \multicolumn{10}{|l|}{ Gastrointestinal disorders ( $n$ ) } \\
\hline Diarrhoea & 4 & 2 & 0 & 4 & 0 & 0 & 8 & 2 & 0 \\
\hline Vomiting & 2 & 0 & 0 & 3 & 0 & 0 & 5 & 0 & 0 \\
\hline Nausea & 2 & 0 & 0 & 2 & 0 & 0 & 4 & 0 & 0 \\
\hline Stomatitis & 1 & 0 & 0 & 2 & 0 & 0 & 3 & 0 & 0 \\
\hline \multicolumn{10}{|c|}{ Skin and subcutaneous tissue disorders $(n)$} \\
\hline Rash & 4 & 0 & 0 & 4 & 0 & 0 & 8 & 0 & 0 \\
\hline Dermatitis acneiform & 1 & 0 & 0 & 1 & 0 & 0 & 2 & 0 & 0 \\
\hline \multicolumn{10}{|c|}{ General disorders and administration site conditions ( $n$ ) } \\
\hline Asthenia & 4 & 0 & 0 & 2 & 0 & 0 & 6 & 0 & 0 \\
\hline Mucositis & 1 & 1 & 0 & 0 & 2 & 0 & 1 & 3 & 0 \\
\hline \multicolumn{10}{|l|}{ Eye disorders $(n)$} \\
\hline Serous retinal detachment & 5 & 0 & 0 & 1 & 0 & 0 & 6 & 0 & 0 \\
\hline \multicolumn{10}{|c|}{ Blood and lymphatic system disorders $(n)$} \\
\hline Neutropenia & 0 & 0 & 0 & 0 & 1 & 2 & 0 & 1 & 2 \\
\hline \multicolumn{10}{|l|}{ Metabolism and nutrition disorders ( $n$ ) } \\
\hline Hypomagnesaemia & 1 & 1 & 0 & 0 & 0 & 0 & 1 & 1 & 0 \\
\hline
\end{tabular}


Table 4. Pharmacokinetics of pimasertib

\begin{tabular}{|c|c|c|c|c|}
\hline & \multicolumn{2}{|c|}{$45 \mathrm{mg}$ per day } & \multicolumn{2}{|c|}{$60 \mathrm{mg}$ per day } \\
\hline & $\begin{array}{l}\text { Day } 1 \\
\text { (test) }\end{array}$ & $\begin{array}{c}\text { Day } 8 \\
\text { (reference) }\end{array}$ & $\begin{array}{l}\text { Day } 1 \\
\text { (test) }\end{array}$ & $\begin{array}{c}\text { Day } 8 \\
\text { (reference) }\end{array}$ \\
\hline \multicolumn{5}{|c|}{$C_{\max }\left(\mathrm{ng} \mathrm{ml}^{-1}\right)$} \\
\hline$N$ & 9 & 9 & 6 & 6 \\
\hline Median & 145 & 196 & 386 & 332 \\
\hline Range & $82.3-418$ & $71.6-660$ & $163-516$ & $214-638$ \\
\hline \multicolumn{5}{|c|}{$T_{\max }(\mathrm{h})$} \\
\hline$N$ & 9 & 9 & 6 & 6 \\
\hline Median & 2 & 1.5 & 0.79 & 1.25 \\
\hline Range & $1.00-6.00$ & $0.53-2.00$ & $0.50-4.00$ & $0.50-2.50$ \\
\hline \multicolumn{5}{|c|}{$\mathrm{AUC}_{(0-\mathrm{t})}\left(\mathrm{ng} \mathrm{ml}^{-1} \cdot \mathrm{h}\right)$} \\
\hline$N$ & 9 & 8 & 5 & 5 \\
\hline Median & 1120 & 1270 & 1390 & 1260 \\
\hline Range & $194-1860$ & 190-2030 & $666-2190$ & $858-2060$ \\
\hline \multicolumn{5}{|c|}{$\mathrm{AUC}_{(0-\infty)}\left(\mathrm{ng} \mathrm{ml}^{-1} \cdot \mathrm{h}\right)$} \\
\hline$N$ & 7 & 8 & 5 & 5 \\
\hline Median & 1160 & 1340 & 1430 & 1340 \\
\hline Range & $226-1950$ & $244-2140$ & $673-2310$ & $869-2170$ \\
\hline \multicolumn{5}{|l|}{$T_{1 / 2}(\mathrm{~h})$} \\
\hline$N$ & 7 & 8 & 5 & 5 \\
\hline Median & 5.31 & 5.12 & 5.56 & 5.08 \\
\hline Range & $2.37-5.97$ & $3.88-7.27$ & $3.53-6.56$ & $4.27-6.25$ \\
\hline \multicolumn{5}{|c|}{$\mathrm{CL} / \mathrm{f}\left(\mathrm{Ih}^{-1}\right)$} \\
\hline$N$ & 7 & 8 & 5 & 5 \\
\hline Median & 38.7 & 34.2 & 42.1 & 44.6 \\
\hline Range & 23.1-199 & $21.0-184$ & $25.9-89.1$ & $27.6-69.1$ \\
\hline \multicolumn{5}{|l|}{$V z / f(I)$} \\
\hline$N$ & 7 & 8 & 5 & 5 \\
\hline Median & 276 & 265 & 348 & 395 \\
\hline Range & $197-680$ & 189-1030 & $210-570$ & $246-425$ \\
\hline \multicolumn{5}{|c|}{ Ratio of $C_{\max }$ test/reference } \\
\hline$N$ & & 8 & & 5 \\
\hline Median & & 0.837 & & 0.751 \\
\hline Range & & $0.345-1.18$ & & $0.359-2.22$ \\
\hline \multicolumn{5}{|c|}{ Ratio of $\mathrm{AUC}_{(0-\infty)}$ test/reference } \\
\hline$N$ & & 7 & & 4 \\
\hline Median & & 0.917 & & 0.825 \\
\hline Range & & $0.738-1.22$ & & $0.759-1.21$ \\
\hline
\end{tabular}

Abbreviations: $\mathrm{AUC}_{0-t}=$ area under the concentration-time curve from time zero to the time of last observation; $\mathrm{AUCO}-\infty=$ area under the concentration-time curve from time zero to infinity; $\mathrm{CL} / \mathrm{f}=$ apparent oral clearance; $C_{\max }=$ maximal concentration; FOLFIRI $=5$ fluorouracil/folinic acid/irinotecan; $T_{\max }=$ time of maximum concentration; $t_{1 / 2}=$ apparent half-life; $V z / f=$ apparent oral volume of distribution. Pharmacokinetics of pimasertib following single 45 and $60 \mathrm{mg}$ doses on day 1 administered concomitantly with FOLFIRI (test) and following single 45 and $60 \mathrm{mg}$ doses on day 8 administered alone (reference).

to $4 \mathrm{~h}$ followed by a slower elimination phase. Median apparent oral clearance $(\mathrm{CL} / f)$ of pimasertib was 34.2 and $44.61 \mathrm{~h}^{-1}$ in the two cohorts. The median apparent volume of distribution $\left(V_{\mathrm{z}} / f\right)$ largely exceeded total body water, at 265 and $395 \mathrm{l}$, respectively. The median apparent pimasertib half-life $\left(t_{1 / 2}\right)$ of pimasertib was $\sim 5 \mathrm{~h}$ in both cohorts (Table 4). The PK of pimasertib administered to colorectal cancer patients at these two dose levels were consistent with data from the first-in-human (FIH) trial IMP28062 of pimasertib monotherapy in advanced solid tumour patients (Houédé et al, 2012).

The observed concentration-time profiles for pimasertib were consistent in the absence and presence of FOLFIRI (Figure 1A). In the presence of FOLFIRI, pimasertib maximum plasma concentrations $\left(C_{\max }\right)$ were reduced by $16.3 \%$ (range: $0.35-1.18$-fold) and pimasertib exposure $\left(\mathrm{AUC}_{0-\infty}\right)$ was reduced by $8.3 \%$ (range: $0.74-$ 1.22 -fold) in the $45 \mathrm{mg}$ per day cohort. Similar changes were
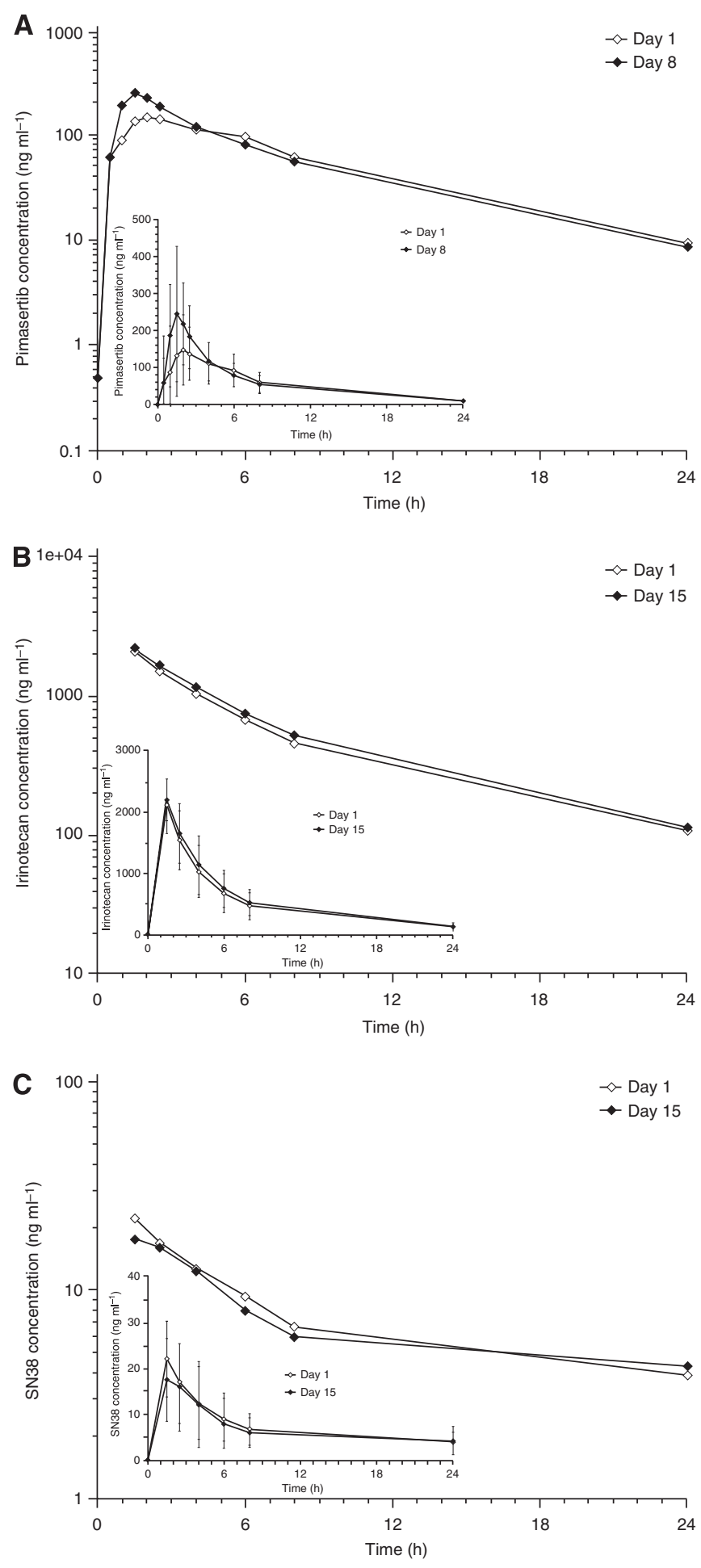

Figure 1. (A) Plasma concentration-time profiles of pimasertib $45 \mathrm{mg}$ per day in the presence (day 1, open diamonds) and absence (day 8, closed diamonds) of FOLFIRI. Data (mean \pm s.d.) are shown as semilog (main figure) and linear (inset) plots. (B) Plasma concentration-time profiles of irinotecan in the presence (day 1, open diamonds) and absence (day 15 , closed diamonds) of pimasertib $45 \mathrm{mg}$ per day. Data (mean \pm s.d.) are shown as semilog (main figure) and linear (inset) plots. (C) Plasma concentration-time profiles of SN-38 in the presence (day 1, open diamonds) and absence (day 15, closed diamonds) of pimasertib $45 \mathrm{mg}$ per day. Data (mean \pm s.d.) are shown as semilog (main figure) and linear (inset) plots. 
Table 5. Pharmacokinetics of irinotecan and SN-38

\begin{tabular}{|c|c|c|c|c|}
\hline & \multicolumn{2}{|c|}{ Irinotecan } & \multicolumn{2}{|c|}{ SN-38 } \\
\hline & $\begin{array}{l}\text { Day } 1 \\
\text { (test) }\end{array}$ & $\begin{array}{c}\text { Day } 15 \\
\text { (reference) }\end{array}$ & $\begin{array}{l}\text { Day } 1 \\
\text { (test) }\end{array}$ & $\begin{array}{c}\text { Day } 15 \\
\text { (reference) }\end{array}$ \\
\hline \multicolumn{5}{|c|}{$C_{\max }\left(\mathrm{ng} \mathrm{ml}^{-1}\right)$} \\
\hline$N$ & 10 & 8 & 10 & 8 \\
\hline Median & 2070 & 2120 & 20.4 & 15.1 \\
\hline Range & $1590-3060$ & $1760-2840$ & $11.4-37.6$ & $9.28-37.5$ \\
\hline \multicolumn{5}{|c|}{$T_{\max }(h)$} \\
\hline$N$ & 10 & 8 & 10 & 8 \\
\hline Median & 1.5 & 1.54 & 1.5 & 1.6 \\
\hline Range & $1.25-2.50$ & $1.50-2.50$ & $1.50-2.25$ & $1.50-2.50$ \\
\hline \multicolumn{5}{|c|}{$\mathrm{AUC}_{(\mathrm{O}-\mathrm{t})}\left(\mathrm{ng} \mathrm{ml}^{-1} \cdot \mathrm{h}\right)$} \\
\hline$N$ & \begin{tabular}{l|}
10 \\
\end{tabular} & 8 & 10 & 8 \\
\hline Median & 10500 & 12700 & 150 & 146 \\
\hline Range & $7130-18500$ & 7040-17900 & $35.4-380$ & $42.5-380$ \\
\hline \multicolumn{5}{|c|}{$\mathrm{AUC}_{(0-\infty)}\left(\mathrm{ng} \mathrm{ml}^{-1} \cdot \mathrm{h}\right)$} \\
\hline$N$ & 9 & 8 & 10 & 7 \\
\hline Median & 10600 & 13600 & 198 & 206 \\
\hline Range & $7650-20600$ & $7330-20400$ & $102-550$ & $64.7-508$ \\
\hline \multicolumn{5}{|l|}{$T_{1 / 2}(\mathrm{~h})$} \\
\hline$N$ & 9 & 8 & 10 & 7 \\
\hline Median & 6.43 & 6.21 & 13.6 & 15.7 \\
\hline Range & $5.32-7.63$ & $5.54-8.77$ & $7.10-21.5$ & $4.63-34.2$ \\
\hline \multicolumn{5}{|c|}{$\mathrm{CL} / \mathrm{f}\left(\mathrm{Ih}^{-1}\right)$} \\
\hline N & 9 & 8 & & \\
\hline Median & 29.4 & 23.5 & NC & NC \\
\hline Range & $16.4-47.0$ & $15.6-46.8$ & & \\
\hline \multicolumn{5}{|l|}{$V z / f(I)$} \\
\hline$N$ & 9 & 8 & & \\
\hline Median & 297 & 212 & NC & NC \\
\hline Range & $147-455$ & $143-382$ & & \\
\hline \multicolumn{5}{|c|}{ Ratio of $C_{\max }$ test/reference } \\
\hline$N$ & & 8 & & 8 \\
\hline Median & & 0.973 & & 1.11 \\
\hline Range & & $0.623-1.13$ & & $0.912-2.04$ \\
\hline \multicolumn{5}{|c|}{ Ratio of $\mathrm{AUC}_{(0-\infty)}$ test/reference } \\
\hline$N$ & & 8 & & 7 \\
\hline Median & & 0.911 & & 0.91 \\
\hline Range & & $0.577-1.08$ & & $0.825-1.58$ \\
\hline
\end{tabular}

Abbreviations: $\mathrm{AUC}_{0-\mathrm{t}}=$ area under the concentration-time curve from time zero to the time of last observation; $\mathrm{AUC}_{0-\infty}=$ area under the concentration-time curve from time zero to infinity; $C L / f=$ apparent oral clearance; $C_{\max }=$ maximal concentration; $N C=$ not calculated; $t_{1 / 2}=$ apparent half-life; $T_{\max }=$ time of maximum concentration; $V z / f=$ apparent oral volume of distribution. Pharmacokinetics of irinotecan and its active metabolite SN-38 following single $180 \mathrm{mg} \mathrm{m}^{-2}$ doses on day 1 administered concomitantly with pimasertib (test) and following single $180 \mathrm{mg} \mathrm{m}^{-2}$ doses on day 15 administered alone (reference).

observed in the $60 \mathrm{mg}$ per day cohort (Table 4). These results indicate that the concomitant administration of FOLFIRI had no apparent direct effect on the single-dose PK of pimasertib.

The PK of irinotecan and its active metabolite SN-38 in the absence of pimasertib are shown in Table 5. Irinotecan $C_{\max }$ levels were reached $1.5 \mathrm{~h}$ after the end of the 90 -min infusion. Median irinotecan CL/f was $23.51 \mathrm{~h}^{-1}\left(15.6-46.81 \mathrm{~h}^{-1}\right)$ and median $V_{\mathrm{z}} / f$ was 2121 (range: 143-3821). Irinotecan was eliminated with a median $t_{1 / 2}$ of $6.2 \mathrm{~h}$ (Table 5).

Intraindividual comparisons to elucidate the effect of single pimasertib doses on irinotecan and its active metabolite $\mathrm{SN}-38$ showed that the observed concentration-time profiles for both compounds were consistent in the absence and presence of pimasertib at both dose levels studied (Figures $1 \mathrm{~B}$ and $\mathrm{C}$ ). In the $45 \mathrm{mg}$ per day pimasertib cohort, irinotecan $C_{\max }$ values were reduced by $2.7 \%$ (range: $0.62-1.13$-fold) and irinotecan exposure $\left(\mathrm{AUC}_{0-\infty}\right)$ was reduced by $8.9 \%$ (range: $0.58-1.08$-fold). Similar changes were observed in the $60 \mathrm{mg}$ per day cohort (Table 5). Formation of irinotecan's active metabolite SN-38 was not affected by the coadministration of pimasertib, as evidenced by an increase in SN-38 $C_{\max }$ values of $11 \%$ (range: $0.912-2.04$-fold). Elimination of SN-38 was also not impacted by concomitant pimasertib administration, as evidenced by comparable $t_{1 / 2}$ values and a decrease of $\mathrm{SN}$ 38 exposure $\left(\mathrm{AUC}_{0-\infty}\right)$ of $9 \%$ (range: $0.83-1.58$ ). The data suggest that single pimasertib doses do not have a direct effect on singledose PK of irinotecan and the formation/elimination of its active metabolite SN-38.

Median intraindividual ratios of 5-FU concentrations in the presence and absence of pimasertib were $0.86,0.59$ and 0.92 at 6,8 and $24 \mathrm{~h}$ postinfusion start. The data suggest that the administration of pimasertib did not have an apparent influence on 5-FU steady-state levels.

\section{DISCUSSION}

In this safety run-in part of a phase II study of second-line treatment in patients with KRAS mt mCRC, dose escalation of pimasertib from 45 to $60 \mathrm{mg}$ per day in combination with FOLFIRI was limited by toxicity (mucositis). Two patients experienced a DLT at the pimasertib $60 \mathrm{mg}$ per day dose level and therefore the MTD of pimasertib in this treatment regimen was considered to be $45 \mathrm{mg}$ per day, the starting dose level for the dose escalation part of this trial. There was one additional DLT (grade 3 hyponatraemia) among the additional six patients in the expanded MTD cohort.

At the MTD of pimasertib $45 \mathrm{mg}$ per day in combination with FOLFIRI, pimasertib was adequately tolerated in patients with mCRC, and no unexpected or new safety signals or concerns were identified. The most frequent TEAEs during the study were diarrhoea and gastrointestinal events. The other frequently reported grade 1 or 2 events were skin rash, asthenia and SRD. The most frequent grade 3 or 4 events were mucosal inflammation and neutropenia. Grade 3/4 mucosal inflammation and neutropenia were not observed as TEAEs in pimasertib monotherapy trials in patients with advanced solid tumours or haematological malignancies (Ravandi et al, 2011; Awada et al, 2012), and thus these TEAEs can be attributed to the combination of FOLFIRI with pimasertib.

Ocular toxicity is a common feature of most MEK inhibitors (Renouf et al, 2012). A total of seven patients reported ocular AEs, none of which were serious. All events were of grade 1 or 2 and all resolved within 3-139 days from the day of onset, except for SRD in two patients. The nature, incidence and severity of ocular AEs were similar to that reported in a previous monotherapy trial of pimasertib in patients with advanced solid tumours (Awada et al, 2012).

In FIH trials of combination therapies, it is important to control patient exposure to individual drugs. This allows elucidation of PK drug-drug interactions that could lead to altered dose-toxicity relationships when drugs are used in combination, potentially requiring changes to the dose-escalation scheme. In this study, coadministration of FOLFIRI did not affect the PK of single-dose pimasertib. Likewise, single doses of pimasertib of 45 and $60 \mathrm{mg}$ per day influenced the PK of neither irinotecan nor its active metabolite/SN-38, indicating that pimasertib does not exert a direct effect on the enzymes involved in the elimination of irinotecan, that is, CYP3A4 and UGT1A1, or human carboxylesterase HCE-2, which catalyses the formation of SN-38. In addition, pimasertib had no apparent effect on steady-state 5-FU levels. This corroborates in vitro data showing that pimasertib does not alter 5-FU metabolism in human liver microsomes (Merck, data on file). It needs to be noted that the PK evaluations in the present study were limited to two doses of pimasertib in a small number of subjects and were not statistically powered to observe predefined 
differences in exposure. This limitation was due to the nature of the study being a FIH trial. The intraindividual crossover analysis implemented in this study to elucidate pimasertib's effects on the PK of irinotecan/SN-38 and 5-FU, as well as effects of FOLFIRI on the $\mathrm{PK}$ of pimasertib in expanded cohorts, however, provides higher statistical power because interindividual variability in the PK of the studied agents can be ignored.

The majority of patients experienced SD (nine patients) and two patients had PR as their best overall tumour response. Furthermore, for six patients with SD as their best response, the SD was maintained for at least 12 weeks. The best overall response spectrum did not seem to differ much between the two pimasertib doses (45 or $60 \mathrm{mg}$ per day) combined with FOLFIRI, suggesting antitumour activity at both doses. However, the estimated probability of observing significant clinical benefit with the combination of pimasertib $45 \mathrm{mg}$ per day combined with FOLFIRI compared with FOLFIRI alone was considered to be low. Thus, the decision was made not to perform the randomised, placebocontrolled phase II part of the study.

The optimal approach to targeting the MAPK pathway in colorectal cancer is not yet established. Further studies are required to determine the most effective regimens for agents such as MEK1/2 inhibitors (as monotherapy and/or in combination with both established and emerging therapies) (Troiani et al, 2014) as well as identifying the patient populations most likely to benefit from such interventions (Chapman and Miner, 2011).

In conclusion, in combination with FOLFIRI, dose escalation of the MEK1/2 inhibitor pimasertib from 45 to $60 \mathrm{mg}$ per day was limited by toxicity. Progression to phase II was not recommended because the potential activity of pimasertib $45 \mathrm{mg}$ per day, administered intermittently, with FOLFIRI was not considered to be sufficient. Alterations in the PK of pimasertib, irinotecan/SN-38 and 5-FU can be excluded as the underlying reason for intolerability of the combination.

\section{ACKNOWLEDGEMENTS}

We thank Frank Campana, MD, for his contribution (writing the protocol, regularly liaising with the clinicians, reviewing and cleaning part of the database) in the study. The study was sponsored by Merck Serono SA, Geneva, an affiliate of Merck $\mathrm{KGaA}$, Darmstadt, Germany. We are fully responsible for the content and editorial decisions for this manuscript. George Xinarianos of Cancer Communications and Consultancy Ltd (Knutsford, UK) provided medical writing services on behalf of the authors, funded by the study sponsor. These included initial drafting of the manuscript and subsequent revision according to guidance from the authors. The final version of the article has been approved by all authors and by the study sponsor.

\section{CONFLICT OF INTEREST}

AC has served as an advisory board member and has received speaker's fees from Merck Serono. JT has served as a consultant and advisory board member for Amgen, Imclone, Lilly, Merck KGaA, Millenium, Novartis, Roche, Sanofi, Celgene, Chugai and Taiho. EVC has received research funding from Merck KGaA paid to institution. ST has received speaker's fees from Merck Serono. OvR is an employee of Merck Serono. BL was an employee of Merck Serono and is currently a non-exclusive consultant for Merck KGaA. VJ is an employee of Cytel Inc, a CRO contracted by Merck KGaA. FC has served as an advisory board member for Merck Serono, Bayer, Astellas and Roche and has received research funding from Merck Serono, Bayer and AstraZeneca. TM, SR, HP, EM and TT declare no conflict of interest.

\section{REFERENCES}

Awada A, Delord JP, Houede N, Lebbe C, Lesimple T, Schellens JHM, Rottey S, Kefford R, Rejeb N, Raymond E (2012) Safety and recommended phase II dose (RP2D) of the selective oral MEK1/2 inhibitor pimasertib (MSC1936369B/AS703026): results of a phase I trial. Eur J Cancer 48(Suppl 6): 185-186 (abstract 604).

Bokemeyer C, Bondarenko I, Makhson A, Hartmann JT, Aparicio J, de Braud F, Donea S, Ludwig H, Schuch G, Stroh C, Loos AH, Zubel A, Koralewski P (2009) Fluorouracil, leucovorin, and oxaliplatin with and without cetuximab in the first-line treatment of metastatic colorectal cancer. J Clin Oncol 27(5): $663-671$.

Bokemeyer C, Van Cutsem E, Rougier P, Ciardiello F, Heeger S, Schlichting M, Celik I, Kohne CH (2012) Addition of cetuximab to chemotherapy as firstline treatment for KRAS wild-type metastatic colorectal cancer: pooled analysis of the CRYSTAL and OPUS randomised clinical trials. Eur J Cancer 48(10): 1466-1475.

Chapman MS, Miner JN (2011) Novel mitogen-activated protein kinase kinase inhibitors. Expert Opin Invest Drugs 20(2): 209-220.

Clark AM, Ma J, Qiu D, Lin J, Syed S, Romanelli A, Spooner E, Shaw J, Rocha C, Tian H (2009) Pharmacokinetics, efficacy and target pathway inhibition in vivo of AS703026, a small molecule inhibitor of MEK. Cancer Res 69(Suppl 9): 3694.

De Roock W, Claes B, Bernasconi D, De Schutter J, Biesmans B, Fountzilas G, Kalogeras KT, Kotoula V, Papamichael D, Laurent-Puig P, Penault-Llorca F, Rougier P, Vincenzi B, Santini D, Tonini G, Cappuzzo F, Frattini M, Molinari F, Saletti P, De Dosso S, Martini M, Bardelli A, Siena S, SartoreBianchi A, Tabernero J, Macarulla T, Di Fiore F, Gangloff AO, Ciardiello F, Pfeiffer P, Qvortrup C, Hansen TP, Van Cutsem E, Piessevaux H, Lambrechts D, Delorenzi M, Tejpar S (2010) Effects of KRAS, BRAF, NRAS, and PIK3CA mutations on the efficacy of cetuximab plus chemotherapy in chemotherapy-refractory metastatic colorectal cancer: a retrospective consortium analysis. Lancet Oncol 11(8): $753-762$.

De Roock W, De Vriendt V, Normanno N, Ciardiello F, Tejpar S (2011) KRAS, BRAF, PIK3CA, and PTEN mutations: implications for targeted therapies in metastatic colorectal cancer. Lancet Oncol 12(6): 594-603.

Delord JP, Houede N, Awada A, Lebbe C, Lesimple T, Schellens JHM, Rottey S, Kefford R, Rejeb N, Raymond E (2012) Pimasertib (MSC1936369B/ AS703026), a selective oral MEK1/2 inhibitor, shows clinical activity in melanoma. Eur J Cancer 48(Suppl 6): 190 (abstract 616).

Douillard JY, Oliner KS, Siena S, Tabernero J, Burkes R, Barugel M, Humblet Y, Bodoky G, Cunningham D, Jassem J, Rivera F, Kocakova I, Ruff P, Blasinska-Morawiec M, Smakal M, Canon JL, Rother M, Williams R, Rong A, Wiezorek J, Sidhu R, Patterson SD (2013) Panitumumab-FOLFOX4 treatment and RAS mutations in colorectal cancer. N Engl J Med 369(11): 1023-1034.

Douillard JY, Siena S, Cassidy J, Tabernero J, Burkes R, Barugel M, Humblet Y, Bodoky G, Cunningham D, Jassem J, Rivera F, Kocakova I, Ruff P, Blasinska-Morawiec M, Smakal M, Canon JL, Rother M, Oliner KS, Wolf M, Gansert J (2010) Randomized, phase III trial of panitumumab with infusional fluorouracil, leucovorin, and oxaliplatin (FOLFOX4) versus FOLFOX4 alone as first-line treatment in patients with previously untreated metastatic colorectal cancer: the PRIME study. J Clin Oncol 28(31): 4697-4705.

Fremin C, Meloche S (2010) From basic research to clinical development of MEK1/2 inhibitors for cancer therapy. J Hematol Oncol 3: 8.

Goutopoulos A, Askew BC, Bankston D, Clark A, Dhanabal M, Dong R, Fischer D, Healey B, Jiang X, Josephson K, Lin J, Ma J, Noonan T, Qiu D, Rocha C, Romanelli A, Shutes A, Spooner E, Tian H (2009) AS703026: a novel allosteric MEK inhibitor. Cancer Res 69(Suppl 9): 4776.

Houédé N, Delord JP, Awada A, Lebbe C, Lesimple T, Schellens JHM, Rottey S, Kefford R, von Richter O, Raymond E (2012) Pharmacokinetics and pharmacodynamics of a selective oral MEK1/2 inhibitor, pimasertib (MSC1936369B/AS703026), in patients with advanced solid tumors. Eur J Cancer 48(Suppl 6): 184 (abstract 600).

Infante JR, Gandhi L, Shapiro G, Burris III HA, Bendell JC, Baselga J, Hsu K, Faivre T, Asatiani E, Heist RS (2012) Phase lb combination trial of a MEK inhibitor, pimasertib (MSC1936369B), and a PI3K/mTOR inhibitor, 
SAR245409, in patients with locally advanced or metastatic solid tumors. J Clin Oncol 30(Suppl): (abstract TPS3118).

Kim K, Kong SY, Fulciniti M, Li X, Song W, Nahar S, Burger P, Rumizen MJ, Podar K, Chauhan D, Hideshima T, Munshi NC, Richardson P, Clark A, Ogden J, Goutopoulos A, Rastelli L, Anderson KC, Tai YT (2010) Blockade of the MEK/ERK signalling cascade by AS703026, a novel selective MEK1/2 inhibitor, induces pleiotropic anti-myeloma activity in vitro and in vivo. Br J Haematol 149(4): 537-549.

Machl AW, Ogden JA, Romanelli A (2009) Efficacy of MEK inhibitor AS703026 in various primary tumor explants. Cancer Res 69(Suppl 9): 3698.

Martinelli E, Troiani T, D'Aiuto E, Morgillo F, Vitagliano D, Capasso A, Costantino S, Ciuffreda LP, Merolla F, Vecchione L, De Vriendt V, Tejpar S, Nappi A, Sforza V, Martini G, Berrino L, De Palma R, Ciardiello F (2013) Antitumor activity of pimasertib, a selective MEK $1 / 2$ inhibitor, in combination with PI $3 \mathrm{~K} / \mathrm{mTOR}$ inhibitors or with multi-targeted kinase inhibitors in pimasertib-resistant human lung and colorectal cancer cells. Int J Cancer 133(9): 2089-2101.

Naing A, Mita M, Komarnitsky P, Milner A, von Richter O, Ogden J, Piha-Paul S, Fu S, Asatiani E, Kurzrock R (2012) Phase I dose-escalation trial of a selective oral MEK1/2 inhibitor, pimasertib (MSC1936369B), combined with an mTOR inhibitor, temsirolimus, in patients with advanced solid tumors. Eur J Cancer 48(Suppl 6): 187 (abstract 608).

Neumann J, Zeindl-Eberhart E, Kirchner T, Jung A (2009) Frequency and type of KRAS mutations in routine diagnostic analysis of metastatic colorectal cancer. Pathol Res Pract 205(12): 858-862.

Prenen H, Tejpar S, Van Cutsem E (2010) New strategies for treatment of KRAS mutant metastatic colorectal cancer. Clin Cancer Res 16(11): 2921-2926.

Ravandi F, Pigneux A, DeAngelo D, Dombret H, Delaunay J, Thomas X, Kadia T, Luepfert C, Asatiani E, Donica M, Kantarjian H, Smith BD (2011) Preliminary clinical, pharmacokinetic (PK) and pharmacodynamic (PD) results of the safety run-in part of a phase II trial of orally available MEKinhibitor MSC1936369 in patients (pts) with advanced hematological malignancies (HM). Blood (ASH Annu Meet Abstr) 118: 21 (abstract 615).

Renouf DJ, Velazquez-Martin JP, Simpson R, Siu LL, Bedard PL (2012) Ocular toxicity of targeted therapies. J Clin Oncol 30(26): 3277-3286.

Santarpia L, Lippman SM, El-Naggar AK (2012) Targeting the MAPK-RAS-RAF signaling pathway in cancer therapy. Expert Opin Ther Targets 16(1): 103-119.

Schmoll HJ, Van Cutsem E, Stein A, Valentini V, Glimelius B, Haustermans K, Nordlinger B, van de Velde CJ, Balmana J, Regula J, Nagtegaal ID, Beets-Tan RG, Arnold D, Ciardiello F, Hoff P, Kerr D, Kohne CH, Labianca R, Price T, Scheithauer W, Sobrero A, Tabernero J, Aderka D,
Barroso S, Bodoky G, Douillard JY, El Ghazaly H, Gallardo J, Garin A, Glynne-Jones R, Jordan K, Meshcheryakov A, Papamichail D, Pfeiffer P, Souglakos I, Turhal S, Cervantes A (2012) ESMO Consensus Guidelines for management of patients with colon and rectal cancer. a personalized approach to clinical decision making. Ann Oncol 23(10): 2479-2516.

Sebolt-Leopold JS (2008) Advances in the development of cancer therapeutics directed against the RAS-mitogen-activated protein kinase pathway. Clin Cancer Res 14(12): 3651-3656.

Troiani T, Napolitano S, Vitagliano D, Morgillo F, Capasso A, Sforza V, Nappi A, Ciardiello D, Ciardiello F, Martinelli E (2014) Primary and acquired resistance of colorectal cancer cells to anti-EGFR antibodies converge on MEK/ERK pathway activation and can be overcome by combined MEK/ EGFR inhibition. Clin Cancer Res 20(14): 3775-3786.

Van Cutsem E, Kohne CH, Hitre E, Zaluski J, Chang Chien CR, Makhson A, D’Haens G, Pinter T, Lim R, Bodoky G, Roh JK, Folprecht G, Ruff P, Stroh C, Tejpar S, Schlichting M, Nippgen J, Rougier P (2009) Cetuximab and chemotherapy as initial treatment for metastatic colorectal cancer. $N$ Engl $J$ Med 360(14): 1408-1417.

Van Cutsem E, Kohne CH, Lang I, Folprecht G, Nowacki MP, Cascinu S, Shchepotin I, Maurel J, Cunningham D, Tejpar S, Schlichting M, Zubel A, Celik I, Rougier P, Ciardiello F (2011) Cetuximab plus irinotecan, fluorouracil, and leucovorin as first-line treatment for metastatic colorectal cancer: updated analysis of overall survival according to tumor KRAS and BRAF mutation status. J Clin Oncol 29(15): 2011-2019.

Van Cutsem E, Nordlinger B, Cervantes A (2010) Advanced colorectal cancer: ESMO Clinical Practice Guidelines for treatment. Ann Oncol 21(Suppl 5): v93-v97.

Ye LC, Liu TS, Ren L, Wei Y, Zhu DX, Zai SY, Ye QH, Yu Y, Xu B, Qin XY, Xu J (2013) Randomized controlled trial of cetuximab plus chemotherapy for patients with KRAS wild-type unresectable colorectal liver-limited metastases. J Clin Oncol 31(16): 1931-1938.

Yoon J, Koo KH, Choi KY (2011) MEK1/2 inhibitors AS703026 and AZD6244 may be potential therapies for KRAS mutated colorectal cancer that is resistant to EGFR monoclonal antibody therapy. Cancer Res 71(2): $445-453$.

This work is published under the standard license to publish agreement. After 12 months the work will become freely available and the license terms will switch to a Creative Commons AttributionNonCommercial-Share Alike 4.0 Unported License. 